\title{
A New Approach for Solving Evolution Problems in Time-Parallel Way
}

\author{
Nabil R. Nassif ${ }^{1}$, Noha Makhoul Karam² ${ }^{2}$ and Yeran Soukiassian ${ }^{3}$ \\ 1 Mathematics Department, \\ American University of Beirut, Beirut Lebanon \\ ${ }^{2}$ IRISA, Campus Beaulieu, \\ Université de Rennes I, Rennes, France \\ ${ }^{3}$ Computer Science Department, \\ American University of Beirut, Beirut Lebanon
}

\begin{abstract}
With the advent of massively parallel computers with thousands of processors, a large amount of work has been done during the last decades in order to enable a more effective use of a higher number of processors, by superposing parallelism in time-domain, even though it is known that time-integration is inherently sequential, to parallelism in the space-domain 8 . Consequently, many families of predictor-corrector methods have been proposed, allowing computing on several time-steps concurrently [5], 6]. The aim of our present work is to develop a new parallel-in-time algorithm for solving evolution problems, based on particularities of a rescaling method that has been developed for solving different types of partial and ordinary differential equations whose solutions have a finite existence time 9 . Such method leads to a sliced-time computing technique used to solve independently rescaled models of the differential equation. The determining factor for convergence of the iterative process are the predicted values at the start of each time slice. These are obtained using "ratio-based" formulae. In this paper we extend successfully this method to reaction diffusion problems of the form $u_{t}=\Delta u^{m}+a u^{p}$, with their solutions having a global existence time when $p \leq m \leq 1$. The resulting algorithm RaPTI provides perfect parallelism, with convergence being reached after few iterations.
\end{abstract}

\section{Introduction}

Modelling complex phenomena in nature and sciences can often lead to solve time-dependent partial differential equations. In that context, and as the timeintegration is inherently sequential, a lot of parallel algorithms have been first developed for the spatial discretization. However, with the advent of massively parallel computers with thousands of processors, the critical mesh resolution often remains far from the high capabilities of these supercomputers as detailed by Farhat and al in [8. And one possible approach, in order to enable a more effective use of a higher number of processors, is to superpose parallelism in timedomain to the parallelism in the space-domain. One of the first (or maybe the first) has been suggested by Nievergelt [1] and led to multiple shooting methods. 
Variants of this method were then developed by several authors, in particular by Chartier and Philippe in [2]. The basic underlining ideas in such algorithms are as follows:

1. Decompose the time integration interval into subintervals (time slices) and predict the initial values of the solution at the beginning of each slice.

2. Solve an initial value problem in each slice, and in a parallel way,

3. Force continuity of the solution branches, at the end points of successive slices, by means of an iterative (for example Newton-like) procedure.

The success of such parallel-time integration would necessarly depend on the number of iterations that should obviously be much less then the number of slices.

More recently, in 2001, Lions, Maday and Turinici proposed in [5] the "parareal algorithm" to solve evolution problems in a time-parallel way. This allows obtaining, in real time and using several processors, solutions that cannot be computed using one processor only. Algorithms has been implemented on linear and nonlinear parabolic problems and approximates successfully the solution later in time before having fully accurate approximations from earlier times. The main features of the method are the following:

1. Choice of a coarse grid that defines "large time-slices".

2. Prediction of the initial values of the solution on each time-slice, using for example a Euler-implicit scheme.

3. Iteration until convergence of the following process:

- Solving independent evolution problems on each time-slice, using any method on a fine grid, leading to discontinuity "jump " between the end value of one slice and the predicted initial value on the next one.

- Correction of the slices initial values by propagation of the jumps,

This scheme is inherently a multi-grid procedure. It combines coarse anf fine resolution in time, in the same spirit as what is done in space for domain decomposition methods, the coarse grid allowing us to propagate very quickly, on the whole interval, information reached on the fine grid up to any desired acuracy. Such algorithm has also received wide attention over the last few years: Maday and Bal proposed in [6] an improved version of the Parareal Algorithm (PA) which gives better answers for nonlinear problems and more importantly allows to tackle non-differentiable problems (whereas the former implementation was based on the linearization of the PDE). Farhat and Chandesris presented in [8] an original mathematical justification to the framework by using the theory of distribution. Many contributions, based on the former works, have been made during the 15th and 16th Domain Decomposition Conferences (Berlin 2003 and New York 2005). We cite mainly the following:

- In 13, Tromeur-Dervout and Guibert introduced adaptativity in the definition of the refinement of the time grid and the time domain splitting (in order to tackle stiff problems), and then proposed an adaptative parallel extrapolation algorithm (for very stiff problems). 
- In [14] Farhat and al proposed a radical change of strategy in order to improve the performance of the parareal algorithm for second-order hyperbolic systems.

In this paper we present a new approach for parallel time integration. Our method is based on a rescaling procedure introduced by Nassif et al in [9] for problems that have finite existence time.

In section 2, we show that such procedure leads to a perfectly parallel-intime alorithm in the case of a scalar ordinary differential equation of the form $y^{\prime}=f(y)$, whereby coarse-grid time-slices can be explicitely found with exact initial predictions at the start of each slice.

In section 3, we generalize this rescaling approach to systems of differential equations that semi-discretize evolution problems of the form:

$$
\frac{\partial u}{\partial t}=\Delta u^{m}+a u^{p} .
$$

This allows predictions of time-slices and simultaneously accurate initial values. We implement this method for linear PDE $(m=p=1)$ getting convergence (on the basis of a fast correcting procedure) in one or two iterations.

\section{Perfect Time-Parallelism for a Scalar ODE}

We consider the case of an elementary differential equation $y^{\prime}=f(y)$ having explosive solution in finite time. One seeks $\left\{y ; T_{b}\right\}, T_{b}<\infty, y:\left[0 ; T_{b}[\rightarrow \mathbb{R}\right.$ such that:

$$
\begin{gathered}
\frac{d y}{d t}=f(y) \text { for } 0<t<T_{b}, \text { with : } \\
y(0)=y_{0}>0, \text { and } \lim _{t \rightarrow T_{b}}|y(t)|=\infty .
\end{gathered}
$$

The function $f$ is such that:

$$
f(y), f^{\prime}(y) \geq 0, f\left(y_{0}\right) \neq 0, \int_{y_{0}}^{\infty} \frac{d y}{f(y)}<\infty .
$$

The Re-scaling method consists in simultaneously introducing change of variables for the time $t$ and the solution $y(t)$ in order to generate a sequence of slices of time intervals. On each of these subintervals, the computation of the solution is controlled by a preset threshold (or cut-off) value $S$. The change of variables is given by:

$$
t=T_{n-1}+\beta_{n} s, y(t)=y_{n-1}+\alpha_{n} z(s)
$$

where $y_{n}=y\left(T_{n}\right)$, and $\left\{\alpha_{n}\right\}$ and $\left\{\beta_{n}\right\}$ are sequences of normalizing parameters characteristic to the re-scaling method. The resolution of the initial problem (1) is then equivalent to that of rescaled models on each of time-slice, $\left[T_{n-1}, T_{n}\right]$, whereby we seek the pair $\left\{z,\left\{s_{n}\right\}\right\}$ where $z:\left[0 ; s_{n}\right] \rightarrow \mathbb{R}$, such that:

$$
\frac{d z}{d s}=g_{n}(z)=\frac{\beta_{n}}{\alpha_{n}} f\left(y_{n-1}+\alpha_{n} z\right), 0<s<s_{n},
$$




$$
z(0)=0, z\left(s_{n}\right)=S \text {. }
$$

The criterion for limiting $z$ not to out-grow pre-set threshold value $S$ is only due to the nature of the solution which in this case is explosive. This condition must be adapted to other cases where the solution has a global existence time taking into account its behavior: bounded, unbounded, oscillatory, etc. Thus, in this particular case where the behavior is explosive, the choice of the sequence $\left\{\alpha_{n}\right\}$ is determined by $\lim _{n \rightarrow \infty} y_{n}=\infty$ and $\lim _{n \rightarrow \infty} T_{n}=T_{b}$, implying the consequent pertinent choices for $\left\{\alpha_{n}\right\}$, specifically: $\alpha_{n}=y_{n-1}$ when $f(y)=O\left(y^{p}\right)$ and $\alpha_{n}=1$ when $f(y)=O\left(e^{y}\right)$. The underlying theory behind these choices is well detailed in [9] and [10]. The sequence $\left\{\beta_{n}\right\}$ is determined in order that the rescaled models (4) verify a self-similarity concept, formal or numerical. This leads into imposing $g_{n}(0)=g_{1}(0), \forall n>1$, implying for $\left\{\beta_{n}\right\}$, the choices: $\beta_{n}=\frac{\alpha_{n}}{f\left(y_{n-1}\right)}$.

Perfect time-parallelism. Use of the re-scaling method for the purpose of parallel-time integration is based on the following principles:

1. The starting values of the solution on each time-slice are exactly determined by:

$$
\forall n, y_{n}=y_{n-1}+\alpha_{n} S, \alpha_{n}=y_{n-1} \text {, or } 1 \text {. }
$$

2. The size of the $n^{t h}$ time slice $\left[T_{n-1}, T_{n}\right]$, is given by: $T_{n}-T_{n-1}=\beta_{n} s_{n}$, with the values of $\beta_{n}$ also pre-determined by $\beta_{n}=\frac{\alpha_{n}}{f\left(y_{n-1}\right)}$ and each $s_{n}$, computed independently of any other, given that the stopping criteria on each slice is the same one and in this scalar case is a function of $S$ only.

Implementation of this method, with analysis of acceleration factors can be found in [15].

\section{Extension to Partial Differential Equations}

We first start by extending the re-scaling method to partial differential equations. This is illustrated on the case of finding $u:[0, T] \times \Omega \rightarrow \mathbb{R}, \Omega \subset \mathbb{R}^{d}, d=1$ or 2, such that:

$$
u_{t}-\Delta u^{m}=a u^{p}
$$

for $m \leq p \leq 1$, homogeneous Dirichlet boundary conditions $(u(x, t)=0, x \in$ $\partial \Omega, t>0)$ and initial conditions $\left(u(x, 0)=u_{0}(x)>0\right)$ allowing the solution to have various behaviors with respect to time $([7])$. A first step consists in changing the variable $u$. Letting $v=u^{m}$ and $q=1 / m$, one has:

$$
\frac{\partial v}{\partial t}=\frac{1}{q v^{q-1}} \Delta v+\frac{a}{q} v^{p q-q+1}, x \in \Omega \subset \mathbb{R}^{d}, t>0
$$

with $v$ verifying the same homogeneous Dirichlet boundary condition of $u$ on $\partial \Omega$ and $v(x, 0)=v_{0}=u_{0}^{m}$. Re-scaling necessitates first semi-discretizing (7). In case 
we use Finite-Differences on the operator $\Delta$, this leads to the following system of ordinary differential equations: Find $V:[0, T] \rightarrow \mathbb{R}^{k}$ such that:

$$
\frac{d V}{d t}=-\frac{1}{q} D_{W} A V+F(V),
$$

where $V(0)=V_{0}$ and $V(t)=\left(V_{i}(t)\right)_{i=1,2, \ldots, k} \approx\left(v\left(x_{i}, t\right)_{i=1,2, \ldots, k}\right.$, $F(V)=\frac{a}{q}\left(f\left(V_{i}(t)^{p q-q+1}\right)_{i=1,2, \ldots, k}, W(t)=\left(\frac{1}{\left[V_{i}(t)\right]^{q-1}}\right)_{i=1,2, \ldots, k}\right.$,

$D_{W}=\operatorname{diag}(W) \in \mathbb{R}^{k \times k}$ and $A$ is a sparse symmetric positive matrix that discretizes the $-\Delta$ operator. Using the change of variables:

$$
V(t)=V_{n-1}+D_{\alpha_{n}} Z(s), \text { and } t=T_{n-1}+\beta_{n} s,
$$

where $V_{n-1}=V\left(T_{n-1}\right)$. Solving then the initial value problem (8) is equivalent to solving on each of the intervals $\left[T_{n-1}, T_{n}\right]$ to the rescaled models:

$$
\frac{d Z}{d s}=\beta_{n} D_{\frac{1}{\alpha_{n}}}\left[-\frac{1}{q} D_{W} A V+F(V)\right], 0<s<s_{n}, Z(0)=0,
$$

with the end of the slice $s_{n}$ being determined by a condition depending on the behavior of the solution. For example, in case of explosive (unbounded) behavior, we use $\left\|Z\left(s_{n}\right)\right\|_{\infty}=S$. Otherwise, if $Z(s)$ cannot reach a threshold value $S$, we limit $s_{n}$ to a maximum $\bar{s}, \forall n$. Thus, extending the choices used for $\alpha_{n}$ and $\beta_{n}$ in the scalar case, it has been shown in 7] and [10 that suitable choices are given by $\alpha_{n}=V_{n-1} \in \mathbb{R}^{k}$ and (for the explosive case)

$$
\beta_{n}=\frac{1}{\left\|V_{n-1}^{p q-q}\right\|_{\infty}} \in \mathbb{R}
$$

Parallel-in-time implementation of the re-scaling method. Parallel-time algorithms require being able to determine: (i) the time slices (coarse-grid) and (ii) good predictions for the starting values of the solution at each of the timeslices. In the above scalar case, as the predicted values were the exact ones, parallelism was perfect. At present, the predicted values are only approximate, therefore requiring a correcting procedure that must be iteratively convergent. The basic idea of our predictions is inspired from the scalar case when $f(y)=$ $O\left(y^{p}\right)$ and the sequence $\left\{y_{n}\right\}$ verifies $y_{n}=y_{0}(1+S)^{n}=y_{n-1}(1+S), r=1+S$ being a transition ratio between two successive initial slice-values. This has been generalized in [7] and [10] to the vector case using the corresponding relation derived from the re-scaling method:

$$
\begin{gathered}
V_{n}=V_{n-1}+\operatorname{diag}\left(\alpha_{n}\right) Z\left(s_{n}\right) \text { where } \alpha_{n}=V_{n-1}, \text { i.e. } \\
V_{n}=\operatorname{diag}\left(V_{n-1}\right)\left(e+Z\left(s_{n}\right)\right)=\operatorname{diag}\left(e+Z\left(s_{n}\right)\right) V_{n-1},
\end{gathered}
$$

where $e \in \mathbb{R}^{k}$ is the vector with all of its components equal to 1 . By Defining the "transition" ratio vector:

$$
r_{n}=\left(e+Z\left(s_{n}\right)\right) .
$$


the relation (13) becomes:

$$
V_{n}=\operatorname{diag}\left(r_{n}\right) V_{n-1} \text {. }
$$

It follows from $\left\|Z\left(s_{n}\right)\right\|_{\infty}=S$ that $\left\|r_{n}\right\|_{\infty}=1+S$, which is invariant with respect to $n$ and on the other hand is consistent with the scalar case $(k=1, d=1)$. Thus, it appears that predicting starting values of the solution at each time-slice would result from a-priori information on $r_{n}$. For that purpose, we propose the following new approach to obtain time-parallelism through appropriate predictions of the transition ratio vectors $r_{n}$.

\section{Parallel Algorithm: Ratio-Based Parallel Time Integration (RaPTI)}

1. We run our method sequentially on $n_{s}$ slices by computing the successive exact values of $r_{n}$, referred to as $r_{n}^{e}$, with a local slice computational tolerance $\epsilon_{\text {tol }}^{l}$.

2. On following slices, $n>n_{s}$, statistical estimates techniques based on exact values $r_{n}^{e}\left(n \leq n_{s}\right)$, allow us to predict values of $r_{n}$, denoted by $r_{n}^{p}$.

3. The previous step leads to compute predicted initial values, $V_{n}^{p}=\operatorname{diag}\left(r_{n}^{p}\right)$ $V_{n-1}^{p}$ for $n>n_{s}$. Note that for $n=n_{s}+1, V_{n-1}^{p}=V_{n-1}^{e}$.

4. At this point, parallel computations of the solution can be executed on each $n^{\text {th }}$ slice, $n>n_{s}$, with a local computational tolerance $\epsilon_{\text {tol }}^{l}$, with starting value $V_{n-1}^{p}$ leading to an end value $V_{n}^{c}$. Knowing that the initial value of the $\left(n_{s}+1\right)^{t h}$ slice is exact, $V_{n}^{c}$ is exact for $n=n_{s}+1$.

5. For $n>n_{s}$, we define the sequence $G_{n}$ of gaps at the end of the $n^{\text {th }}$ slice, as $G_{n}=V_{n}^{c}-V_{n}^{p}$.

6. For a given global computational tolerance $\epsilon_{t o l}^{g}>\epsilon_{t o l}^{l}$, we determine $n_{\text {conv }}>n_{s}$ such that for each $n^{\text {th }}$ slice, $n_{s}<n \leq n_{\text {conv }},\left\|G_{n}\right\|_{\infty} \leq \epsilon_{\text {tol }}^{g}$, i.e. convergence is reached up to the $n_{c o n v}^{t h}$ slice.

7. We update $n_{s}$ by $n_{\text {conv }}$ and repeat steps 2 to 6 until the maximum time $T$, defining the interval $[0, T]$ of the evolution problem is reached.

Unlike the Parareal algorithm [5], our RaPTI procedure, exludes any sequential computation for $n>n_{s}$. Furthermore, since this new method is based on rescaling the variables, it generates dynamically the slices which may vary in size depending on the behavior of the solution. Obviously, the success of this method depends on the number of iterations (that repeat steps 2 to 6 ). This number should be much less than the number of slices needed to reach $T$. In fact, our tests have revealed cases of perfect parallelism where convergence has been reached in one or two iterations, particularly when the solution of the diffusion-reaction problem has a global time existence, i.e. $p \leq 1$. When $p>1$, the solution blows up in a finite time and the RaPTI algorithm fails to give fast convergence unless we impose a gross global computational tolerance. A rule of thumb we have used in such situation consists in changing step 7 (correction step) of the algorithm to become:

\section{7'. Repeat steps 2 to 6 using "Lions parareal algorithm"}

This accelerates convergence at the expense of imposing sequential computations for predicting the new corrected values at begining of the slices. 
Tests and Numerical results. Our experiments were run on the following problem:

$$
\begin{cases}\frac{\partial u}{\partial t}-\Delta u=3 u^{p} & \text { for } x \in \Omega=]-1 ; 1[\text { and } t \geq 0 \\ u(x, t)=0 & \text { for } x \in \partial \Omega \text { and } t \geq 0 \\ u(x, 0)=\left(1-x^{2}\right) & \text { for } x \in \Omega\end{cases}
$$

We have used a threshold $S=2$ to determine the sizes of the slices, a discretization space step of $h=1 / 8$, a global tolerance $\epsilon_{t o l}^{g} \geq \epsilon_{\text {tol }}^{l}=5 \times 10^{-8}$ where $\epsilon_{t o l}^{l}$ is the local tolerance used in computing the solution on every slice using a $4^{\text {th }}$ order explicit Runge-Kutta method. Our results are summarized in the following tables where first column indicates the number of slices used for starting sequentially the computations:

1. $p=1 ; \epsilon_{\text {tol }}^{g}=\frac{10^{-7}}{2}$.

\begin{tabular}{|c|c|c|}
\hline$n_{s}$ & Number of Slices & Iterations with RaPTI \\
\hline 2 & 16 & 1 \\
\hline 2 & 32 & 1 \\
\hline 2 & 64 & 1 \\
\hline 2 & 128 & 1 \\
\hline
\end{tabular}

2. $p=0.8 ; \epsilon_{\text {tol }}^{g}=\frac{10^{-7}}{2}$.

\begin{tabular}{|c|c|c|c|}
\hline & & \multicolumn{2}{|c|}{ Number of Iterations } \\
\hline$n_{s}$ & Number of Slices & RaPTI & RaPTI with Parareal for correction (7’) \\
\hline 8 & 16 & 1 & 1 \\
\hline 8 & 32 & 1 & 1 \\
\hline 8 & 64 & 2 & 3 \\
\hline 8 & 128 & 2 & 3 \\
\hline
\end{tabular}

3. $p=1.05$; Results using RaPTI.

\begin{tabular}{|c|c|c|c|c|}
\hline & & \multicolumn{3}{|c|}{ Iterations } \\
\hline$n_{s}$ & Number of Slices & $\epsilon_{\text {tol }}^{g}=\frac{10^{-4}}{2}$ & $\epsilon_{\text {tol }}^{g}=\frac{10^{-3}}{2}$ & $\epsilon_{\text {tol }}^{g}=\frac{10^{-2}}{2}$ \\
\hline 2 & 8 & 5 & 1 & 1 \\
\hline 2 & 16 & 13 & 6 & 1 \\
\hline 2 & 32 & 29 & 22 & 2 \\
\hline
\end{tabular}

4. $p=1.05$; Results with ratio-based predictions and corrections via Lions Parareal scheme.

\begin{tabular}{|c|c|c|c|c|}
\hline & & \multicolumn{3}{|c|}{ Number of Iterations } \\
\hline$n_{s}$ & Number of Slices & $\epsilon_{\text {tol }}^{g}=\frac{10^{-4}}{2}$ & $\epsilon_{\text {tol }}^{g}=\frac{10^{-3}}{2}$ & $\epsilon_{\text {tol }}^{g}=\frac{10^{-2}}{2}$ \\
\hline 2 & 8 & 3 & 1 & 1 \\
\hline 2 & 16 & 3 & 3 & 1 \\
\hline 2 & 32 & 5 & 4 & 3 \\
\hline
\end{tabular}




\section{Perspectives}

We are presently testing the method for non-linear problems $(\forall m, p)$, with the RaPTI algorithm yielding the same super-convergence process when $p \leq m \leq 1$. We are also seeking a new prediction process to handle hyperbolic problems in which the solution exhibits quasi-periodic behavior.

\section{References}

1. J.Nievergelt. Parallel methods for integration ordinary differential equations. Comm. ACM, 7:731-733, 1964

2. P.Chartier, B.Philippe. A parallel shooting technique for solving dissipative $O D E$ 's. Computing, vol.51, n3-4, 1993, p.209-236.

3. J. Erhel, S. Rault. Algorithme parallèle pour le calcul d'orbites. SpringerVerlag, (1989).

4. Fayad D., Nassif N., Cortas M. Rescaling technique for the Numerical computation of blowing-up solutions for semi-linear parabolic equations. 2000,Manuscript.

5. J.L. Lions, Y.Maday, G.Turinici. Résolution d'EDP par un schéma en temps "pararéel". C.R.Acad.Sci.Paris, t.332, Srie 1, p.661-668. 2001.

6. Y. Maday, G. Bal. A parareal time discretization for non-linear pde's with application to the pricing of an American put. Recent developments in Domain Decomposition Methods (Zurich 2002), Lecture Notes in computational Science and Engineering, 23:189-202, Springer 2002.

7. Makhoul-Karam N. Résolution numérique d'équations paraboliques semilinéaires à caractère d'explosion ou d'extinction par des méthodes de redimensionnement. Mémoire de DEA en MSI, 2002-2003.

8. Ch. Farhat, M.Chandesris. Time-decomposed parallel time-integrators. Int. J. Numer. Meth. Engng 2003. 58: 1397-1434.

9. N.R.Nassif, D.Fayad, M.Cortas. Slice-Time Computations with Re-scaling for Blowing-Up Solutions to Initial Value Differential Equations. V.S. Sunderam. et al. (Eds): ICCS 2005, LNCS 3514, pp. 58-65. Springer-Verlag 2005.

10. Cortas M. Méthode de re-dimensionnement (Rescaling technique) pour des équations aux dérivées ordinaires du 1er ordre à caractère explosif. Thèse, Université Bordeaux 1. Janvier 2005.

11. S.Vanderwalle. PARAREAL in a historical perspective (a review of space-time parallel algorithms). In the 16th International Conference on Domain Decomposition Methods, NYU 2005. Proceedings.

12. M.Gander, S.Vanderwalle. On the superlinear and linear convergence of the parareal algorithm. In the 16th International Conference on Domain Decomposition Methods, NYU 2005. Proceedings.

13. D.Guibert, D.Tromeur-Dervout. Adaptative Parareal for systems of ODEs. In the 16th International Conference on Domain Decomposition Methods, NYU 2005. Proceedings.

14. C.Farhat, J.Cortial, H.Bavestello, C.Dastillung. A time-decomposed timeparallel implicit algorithm for accelerating the solution of second-order hyperbolic problems. Berkley-Stanford computational fest, 7 mai 2005.

15. H.Arnaout, H.Mneimneh, Y.Soukiassian Parallel Algorithm for Sliced-Time Computation with Re-scaling for Blowing-up Solutions to Initial Value Problems. Master's Project. American University of Beirut. June 2005. 